\title{
Minimal Neuronal Model for the Transmission Path of Acupuncture Signal
}

\author{
Ying-Mei Qin ${ }^{1}$, Chun-Xiao Han*1, Bei Liu ${ }^{1}$, Yan-Qiu Che ${ }^{1}$ \\ ${ }^{1}$ Tianjin Key Laboratory of Information Sensing and Intelligent Control \\ Tianjin University of Technology and Education \\ Tianjin, China. \\ E-mail: cxhan@tju.edu.cn
}

\begin{abstract}
Acupuncture has been proved to be effective for treatment of diseases, but the underlying mechanisms about acupuncture are still unknown. We design the animal experiment and process the data. We focus on the firing patterns evoked by acupuncture and developed Izhikevich neuronal model to characterize acupuncture signal. It is shown that this minimal model could account for some important experimental phenomena. Computational studies provide more insight in the underlying mechanisms of acupuncture.
\end{abstract}

Keywords-Acupuncture; Izhikevich neuronal model; neural signal.

\section{INTRODUCTION}

Acupuncture is thought to be useful for treatment of diseases, but the potential mechanisms about acupuncture remain unknown [1-4]. Zusanli Point (ZP) has usually been studied by researchers because it is believed to be one of the most useful points in traditional Chinese medicine. So we design the experiment to obtain neural electrical information in spinal dorsal root ganglion (SDRG) by stimulate ZP.

Manual acupuncture with certain frequencies modulates heart rate, and arterial blood pressure and other subjects [5]. Which property of acupuncture plays the key role, frequency or manual pattern? This question is still open. Furthermore, the propagation pathway of electrical information induced by acupuncture have also received little focus in previous researches.

Spike sorting algorithm is a basic tool in neural signals analysis. It has been extensively applied in discussing encoding of external stimuli $[6,7]$. Encode and decode mechanisms of manual acupuncture based on spike sorting algorithm are studied by revealing the various spatiotemporal firing modes [8].

As computational neuroscience plays a more and more important role in understand neural mechanisms, a model of the acupuncture signal propagation pathway based on the feedforward network (FFN) are established [9]. The simulation result is consistent with the experimental results of a phenomenon, such as adaption. However, this feedforward network model is very complicated because it includes synaptic delays and rules of the synaptic plasticity. Besides, it is much easier to study the dynamical characteristics in lowdimensional space. So, it is very important to get a minimal neuronal model of the propagation pathway of acupuncture

This work is supported by National Nature Science Foundation under Grant No.61401312. The Natural Science Foundation of Tianjin (Grant No. 13JCQNJC03700,15JCYBJC19000).We would also acknowledge the support of Tianjin University of Technology and Education (Grant No. KYQD14006, RC14-09, RC14-49, RC14-59) information, which makes it much easier for studying the function mechanism of manual acupuncture.

It has been known that Izhikevich neuronal model reproduced plenty of neuronal dynamical characteristics [10, 11]. Besides that, someone has been used Izhikevich spiking neuron model with dynamic synapse to research the properties of spinal dorsal horn [12]. Therefore, we thought that Izhikevich spiking neuron model is appropriate for establishing the minimal model of the propagation pathway of acupuncture signal.

This paper is structured as follows. In section II, we first described experiment design and signal process. In section III, Izhikevich neuronal model is established to mimic the spiking pattern of acupuncture information. Then, the parameters of the model are modulated to be better consistent with the experimental results. Finally, some discussion and conclusion are proposed.

\section{NEURAL SIGNAL PROCESS}

\section{A. Animal Experiments Design}

We choose the healthy rats as the experimental subjects, and they would be anesthetized before the experiment. Nerve tracts in L4 spinal root are separated to record the neural electrical signal. Lifting-thrusting method ('tb'), which lasts for a minute, is taken at $\mathrm{ZP}$ to study the functions of manual acupuncture with various frequencies.

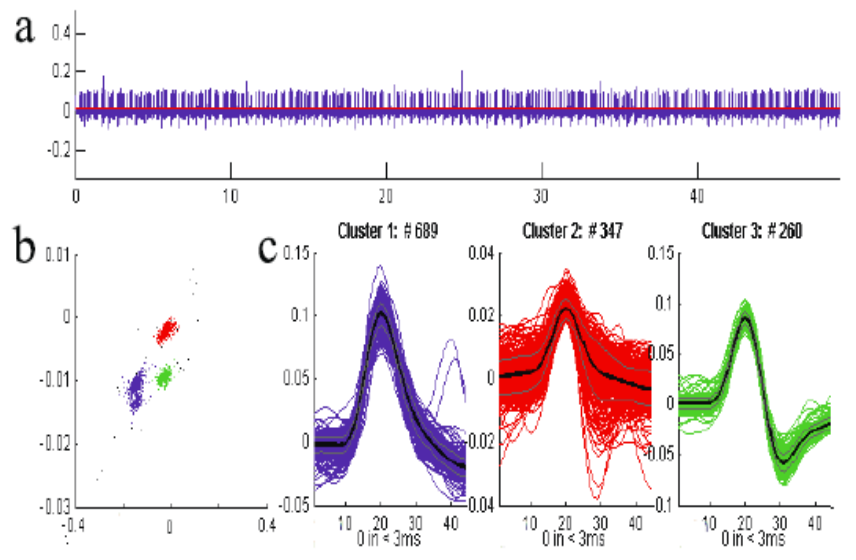

Fig. 1: Process of neural data. (a) The acupuncture signals; (b) feature extraction; (c) Spike patterns. 


\section{B. Analysis of the Spiking Time Series}

The data obtained at SDRG include firing information of large numbers of neurons. We aim to obtain the firing of individual neurons by spike-detecting and sorting method [9]. Different spike shapes are classified based on the wavelet feature extraction as shown in Fig.1.

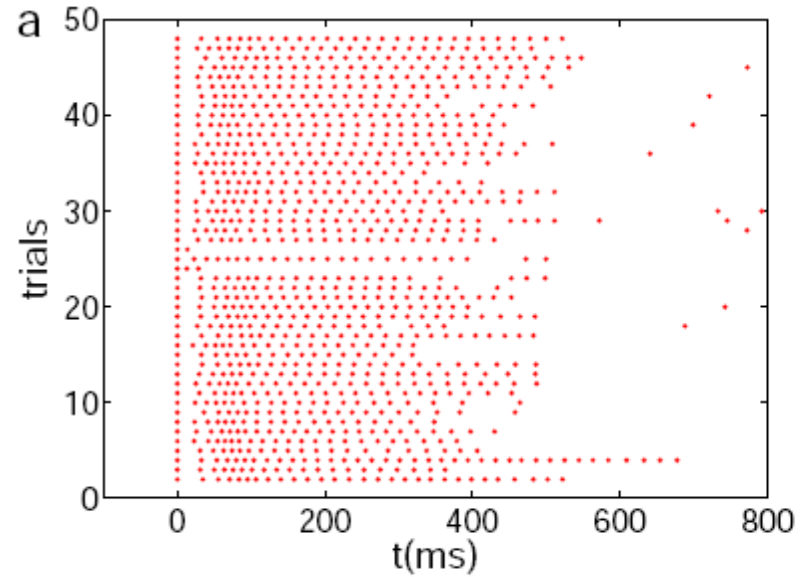

(a)

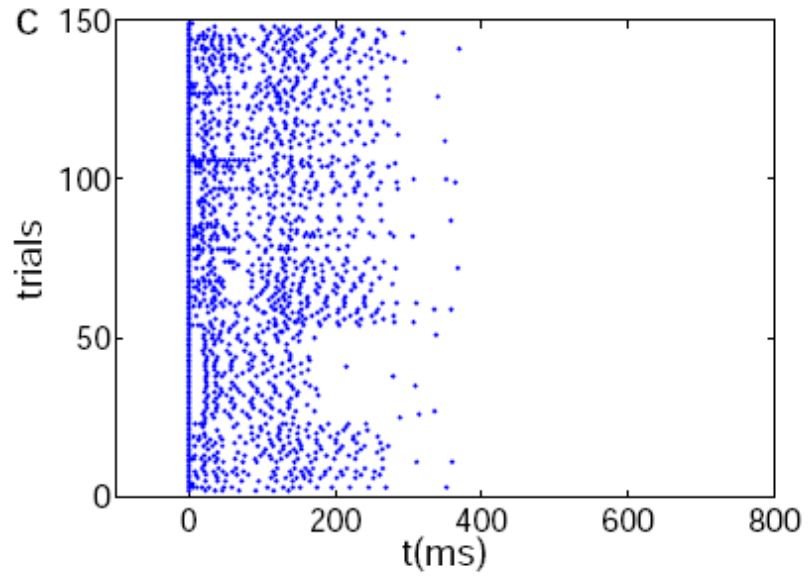

(c)

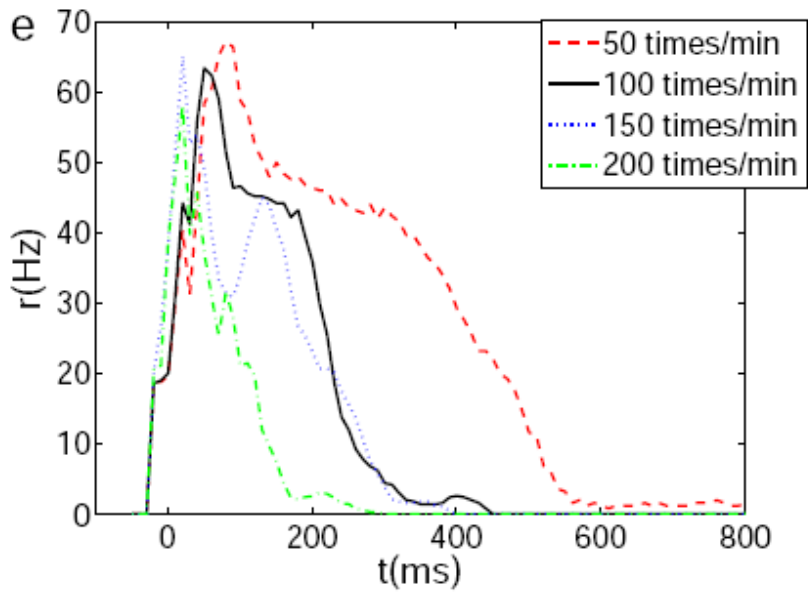

(e)

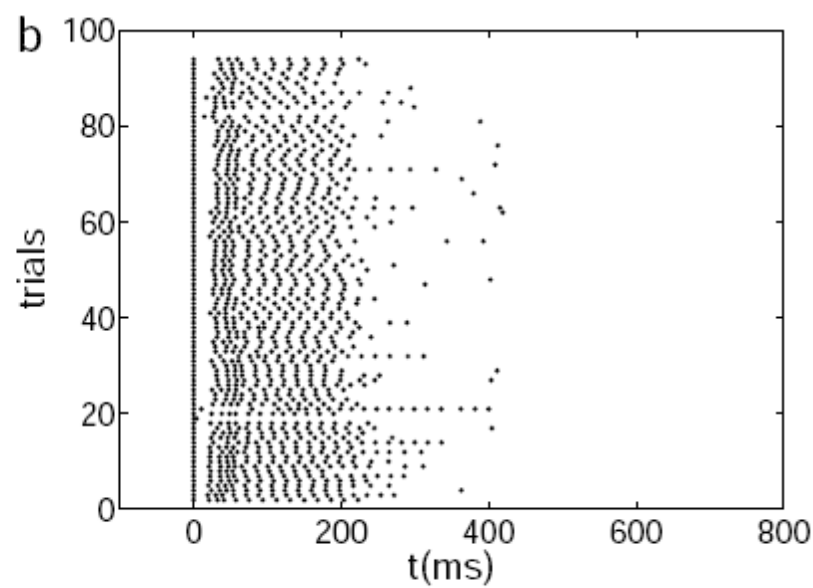

(b)

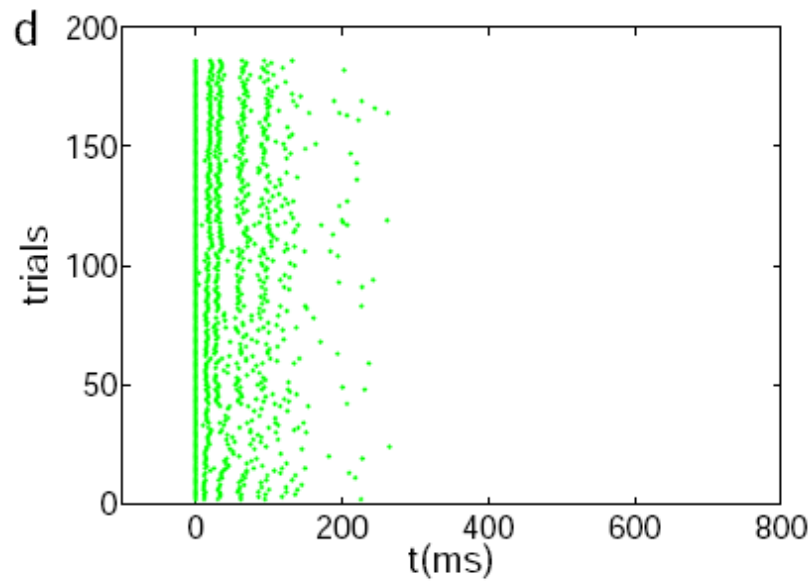

(d)

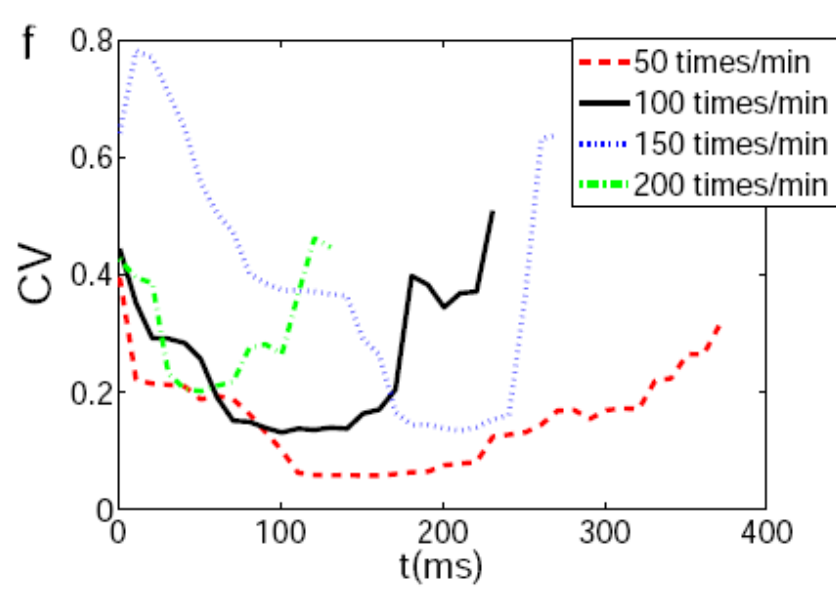

(f)

Fig. 2: Characteristics of 4 different frequencies acupuncture. (a) 50 times/min; (b) 100 times/min; (c) 150 times/min; (d) 200 times/min; (e) PSTHs; (f) Coefficient of variation of inter-spike intervals(ISI). 


\section{Neural Encoding of Acupuncture Stimuli}

Figure 2(a)-(d) show the raster plots of the spike timing of cluster 1 neuron of each experiment. The horizontal axis denotes the time of one trial. Each point shows that there is a spike at the moment. Multiple trials are represented by the vertical axis. PSTHs (Peri-Stimulus-Time Histogram) curves are also calculated.

Different firing patterns are shown in Figure 2(e)(f). The average fire rates first increase and then it gradually reduced to a certain frequency. We could interpret the phenomenon by neuronal adaption in computational neuroscience.

Neural adaption is a physiological phenomenon, where the neuronal firing activity weakens when neurons under a continuing stimulus [13]. From the point of function, adaption is thought to be significant in encoding sensory signals [14][16].

\section{MINIMUM ACUPUNCTURE MODEL}

We could obtain some useful information about manual acupuncture with signal analysis. However, it could not be enough for helping us understand the mechanisms of these physiological phenomenon. So the construction of neuron models is helpful to obtain these characteristics and find the potential mechanisms.

Here, we use the simple spiking model: Izhikevich model [17] to describe the neuron in the spinal dorsal root , as shown in equation (2) and (3),

$$
\left\{\begin{array}{l}
\dot{v}=0.04 v^{2}+5 v+140-u+I+\xi(\mathrm{t}) \\
\dot{u}=a(b v-u)
\end{array}\right.
$$

with resetting rules

$$
\text { if } v \geq 30 \mathrm{mv} \text {, then }\left\{\begin{array}{l}
v \leftarrow c \\
u \leftarrow u+d
\end{array}\right.
$$

$v$ represents the membrane potential of the neuron and $u$ represents a membrane recovery variable. According to equation (3). The model shows many characteristics when we give the model appropriate parameter values [11]. To reproduce the neuronal adaption, we take $(b, c)=(0.2 ;-65)$ and $(a, d)=(0.01 ; 8)$ for the neuron model. In order to depict the differences between trials, we hypothesize that there are disturbances in the acupuncture signal propagation pathway.

This disturbances is represented by the external noise $\xi(\mathrm{t})$, which is thought to be white Gaussian noise with zero mean and power $D$ (i.e., $\left\langle\xi_{i, j}(\mathrm{t}) \xi_{i, j}^{\prime}(\mathrm{t})\right\rangle=2 D \delta\left(\mathrm{t}-\mathrm{t}^{\prime}\right) \quad$ ). When considering the effects of the noises, the model simulation result is more consistent with experimental results.

Fig.3 shows the spike raster plot of Izhikevich neuron model in plenty of trials. Each point of the figure shows a neuronal spike and each line represent a trial. The parameters of Izhikevich model stay the same for each trial and the noises are irrelevant. It can be seen that fire of the neuron from frequently to sparsely as time increasing, which is totally consistent with neuronal adaption. Comparing Fig.3 and Fig.2, it could be found that the firing pattern induced by Izhikevich model is consistent with that evoked by manual acupuncture stimulation.

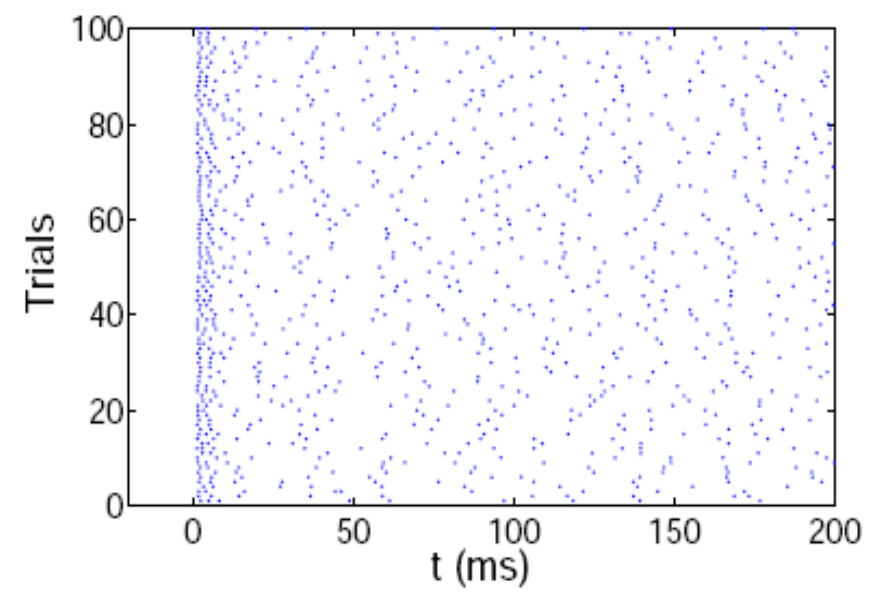

Fig. 3: Raster plots of spikes of Izhikevich model in multiple trials. $D=30 \quad a=0.01 \quad I=30$.

To make our result more quantitative and reproduce the experimental results in detail, we adjust the parameters in the model systematically. The curves of time-dependent firing rate are chosen as the main criterion to measure the similarity between the experimental and computational results. The curves of time-dependent firing rate of Izhikevich model with different parameter $a$ are shown in Fig.4. It is found that the adaption phenomena become obvious as the decrease of $a$. Furthermore, the mean firing rate increase with parameter $a$. After comparing this figure with Fig. 2(e), we found that the simulation result is closer to experimental result when $a=0.012$. It is shown that both the peak value and the firing rate after adaption are very similar between the experiment (especially for experiments of 50 times/min and 100 times/min) and model simulation results. It is implied that Izhikevich model is suitable for modelling the transmission path of acupuncture signal.

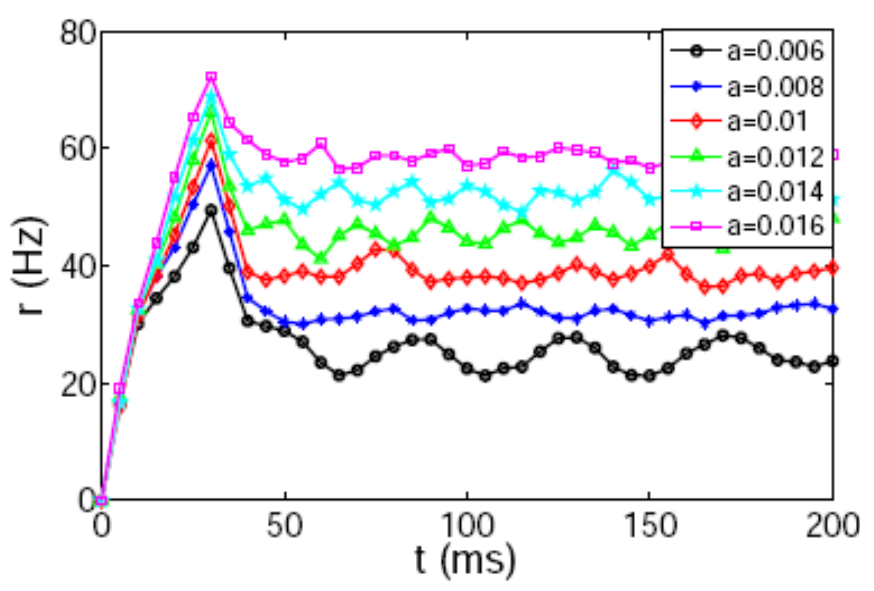

Fig. 4: Time-dependent firing rate of Izhikevich model with different parameters. 


\section{DISCUSSION AND CONCLUSION}

In addition to experimental data analysis of acupuncture signal, we try to establish the minimal neuron model for the acupuncture neurons to make the simulation results accord with experimental results and reproduce the characteristics of bioexperiment signals. Similar firing rate curves are reproduced after the refinement of the parameters in Izhikevich model. We also found that complex network structure and evolution rules are not necessary for the modeling of the acupuncture signal propagation pathway.

There are other advantages of this minimal neuron model. First, the parameters in Izhikevich model are easier to adjust and the results of model are more consistent with the experimental results. Then, we can obtain more complicated model according to this single neuron model. However, there also exist disadvantages for the minimal model. The modeling is according to extract the firing of the main neuron with sorting algorithm, so the firing patterns of multi-neurons could not be forecasted based on this minimal neuron model. This problem could be settled by establishing complex neuronal networks according to Izhikevich model. The constructions of the minimal model compress the parameter space, and make estimation much easier.

\section{REFERENCES}

[1] S. Andersson, T. Lundeberg, Acupuncture - from Empiricism to Science Functional Background to Acupuncture Effects in Pain and Disease, Medical Hypotheses, 45 (1995) 271-281.

[2] T.T.H. Wang, Y. Yuan, Y. Kang, W.L. Yuan, H.T. Zhang, L.Y. Wu Effects of acupuncture on the expression of glial cell line-derived neurotrophic factor (GDNF) and basic fibroblast growth factor (FGF2/bFGF) in the left sixth lumbar dorsal root ganglion following removal of adjacent dorsal root ganglia, Neurosci. Lett., 382 (2005) 236-241.

[3] J.S. Han, Acupuncture: neuropeptide release produced by electrical stimulation of different frequencies, Trends Neurosci., 26 (2003) 17-22.

[4] J. Ezzo, B. Berman, V.A. Hadhazy, A.R. Jadad, L. Lao, B.B. Singh, Is acupuncture effective for the treatment of chronic pain? A systematic review, Pain, 86 (2000) 217-225.

[5] M. Backer, M.G. Hammes, M. Valet, M. Deppe, B. Conrad, T.R. Tolle, G. Dobos, Different modes of manual acupuncture stimulation differentially modulate cerebral blood flow velocity, arterial blood pressure and heart rate in human subjects, Neurosci. Lett., 333 (2002) 203-206.

[6] R.Q. Quiroga, Z. Nadasdy, Y. Ben-Shaul, Unsupervised spike detection and sorting with wavelets and superparamagnetic clustering, Neural Computation, 16 (2004) 1661-1687.

[7] R. Quian Quiroga, S. Panzeri, Extracting information from neuronal populations: information theory and decoding approaches, Nat. Rev. Neurosci., 10 (2009) 173-185.

[8] Men, C., Wang, J., Deng, B., Wei, X.L., Che, Y.Q., Han, C.X. Decoding acupuncture electrical signals in spinal dorsal root ganglion. Neurocomputing, 79(0), (2012): 12-17.

[9] Men, C., Wang, J., Qin, Y.M., Tsang, K.M, B. Deng. Characterizing the transmission of acupuncture signal: A combination of experimental and computational study. Applied Mathematical Modelling, 36(10), (2012): 4742-4749.

[10] Izhikevich, E. M., Gally, J. A., Edelman, G. M. Spike-timing Dynamics of Neuronal Groups. Cerebral Cortex, 14(8), (2004): 933-944.

[11] Izhikevich, E. M. Polychronization: computation with spikes. Neural Comput, 18(2), (2006): 245-282.
[12] Farajidavar, A., Saeb, S., Behbehani, K. Incorporating synaptic timedependent plasticity and dynamic synapse into a computational model of wind-up. Neural Networks, 21(2-3), (2008): 241-249.

[13] Cortes, J., Marinazzo, D., Series, P., Oram, M., Sejnowski, T., Rossum, M. W. The effect of neural adaptation on population coding accuracy. Journal of Computational Neuroscience, 32(3), (2012): 387-402.

[14] Ulanovsky, N., Las, L., Farkas, D., Nelken, I. Multiple time scales of adaptation in auditory cortex neurons. J Neurosci, 24(46), (2004): 10440-10453.

[15] Gutnisky, D. A., Dragoi, V. Adaptive coding of visual information in neural populations. Nature, 452(7184), (2008): 220-224.

[16] Kohn, A. Visual adaptation: physiology, mechanisms, and functional benefits. J Neurophysiol, 97(5), (2007): 3155-3164.

Izhikevich, E. M. Simple model of spiking neurons. IEEE TransNeural Netw, 14(6), (2003): 1569-1572. 